\title{
REMOCIÓN DE NUTRIMENTOS POR FRUTOS DE AGUACATE MÉNDEZ
}

\author{
NUTRIENT REMOVAL BY MENDEZ AVOCADO FRUIT
}

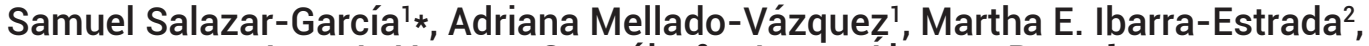 Juan A. Herrera-González ${ }^{3}$ y Arturo Álvarez-Bravo'}

\begin{abstract}
'Instituto Nacional de Investigaciones Forestales, Agrícolas y Pecuarias (INIFAP), Campo Experimental Santiago Ixcuintla, Santiago Ixcuintla, Nayarit, México. ${ }^{2}$ Investigadora Independiente, Santiago Ixcuintla, Nayarit, México. ${ }^{3}$ NIFAP, Campo Experimental Uruapan, Uruapan, Michoacán, México.
\end{abstract}

\section{RESUMEN}

El aguacate (Persea americana Miller) cv. Méndez es de importancia económica en México porque se cosecha cuando baja la producción de Hass, por lo que alcanza mejor precio. Con el fin de conservar esta ventaja comercial se requiere producir frutos de buena calidad mediante la aplicación de los nutrientes requeridos por el cultivo. Como el estado nutrimental de los frutos de aguacate es influenciado por factores edáficos, climáticos, manejo agronómico, fertilización, cultivar y época de floración, que interaccionan en una zona productora, el objetivo de esta investigación fue comparar la concentración y remoción de N, P, K, Ca, Mg, S, Fe, Cu, Mn, Zn y B por los tejidos del fruto (exocarpio o piel, mesocarpio o pulpa, cubierta seminal y embrión) de la producción principal (junio a septiembre) del aguacate Méndez cultivado en las regiones de Jalisco, Michoacán y Nayarit. Se recolectaron frutos por huerto (siete) y región (tres) con $\geq 22.7 \%$ de materia seca en el mesocarpio (método del horno de microondas). De cada tejido del fruto se obtuvo el peso de materia fresca y seca con la finalidad de determinar la concentración de nutrimentos y calcular la remoción por tejido y fruto completo. Se realizó análisis de varianza de una vía y la prueba de comparación de medias WallerDuncan. El exocarpio de los frutos de Jalisco removió más $\mathrm{P}, \mathrm{Ca}$, Fe y $\mathrm{Cu}$. En Jalisco y Michoacán el mesocarpio tuvo mayor remoción de $\mathrm{K}, \mathrm{Mg}$ y Cu. La cubierta seminal de los frutos de Jalisco sobresalió en $\mathrm{K}$, Ca y Cu. En cuanto al embrión, los frutos de Jalisco y Michoacán presentaron la mayor remoción de nutrimentos. En Michoacán y Jalisco los frutos presentaron la mayor remoción total de $\mathrm{P}\left(0.41 \mathrm{a} 0.43 \mathrm{~kg} \mathrm{t}^{-1}\right), \mathrm{K}\left(4.20 \mathrm{a} 4.42 \mathrm{~kg} \mathrm{t}^{-1}\right), \mathrm{Mg}(0.34$ a 0.35 $\left.\mathrm{kg} \mathrm{t}^{-1}\right)$ y Fe $\left(10.18\right.$ a $\left.10.25 \mathrm{~g} \mathrm{t}^{-1}\right)$, mientras que en Nayarit fue el $\mathrm{B}\left(13.25 \mathrm{~g} \mathrm{t}^{-1}\right)$.

Palabras clave: Persea americana Miller, ecofisiología, mesocarpio, nutrición mineral.

\section{SUMMARY}

Avocado (Persea americana Miller) cv. Mendez is of economic importance in Mexico because it is harvested when the production of Hass decreases, so it reaches a better price. In order to preserve this commercial advantage, it is necessary to produce high quality fruit by applying the nutrients required by the plantation. As the nutritional status of avocado fruit is influenced by soil and climatic factors, agronomic management, fertilization, cultivar and flowering season, which interact in a producing area, the aim of this research was to compare the concentration and removal of de $\mathrm{N}, \mathrm{P}, \mathrm{K}, \mathrm{Ca}, \mathrm{Mg}, \mathrm{S}, \mathrm{Fe}, \mathrm{Cu}$, $\mathrm{Mn}, \mathrm{Zn}$ and $\mathrm{B}$ by fruit tissues (exocarp or skin, mesocarp or pulp, seed coat and embryo) of the main crop (June to September) of the Mendez avocado produced in the regions of Jalisco, Michoacan and Nayarit. Fruit was collected by orchard (seven) and region (three) with $\geq 22.7 \%$ of dry matter in the mesocarp (by microwave method). The weight of fresh and dry matter was obtained from tisusue of each fruit to determine the concentration of nutrients and calculate the removal from each tissue and whole fruit. One-way analysis of variance and the Waller-Duncan mean comparison test were performed The exocarp of fruit from Jalisco removed more $\mathrm{P}, \mathrm{Ca}$, Fe and $\mathrm{Cu}$. In Jalisco and Michoacan the mesocarp exerted larger removal of $\mathrm{K}, \mathrm{Mg}$ and $\mathrm{Cu}$. The seed coat of the fruit from Jalisco excelled in $\mathrm{K}, \mathrm{Ca}$ and $\mathrm{Cu}$. As for the embryo, fruit from Jalisco and Michoacan presented the highest removal of nutrients. In Michoacan and Jalisco the fruit showed the largest total removal of $P(0.41$ to $\left.0.43 \mathrm{~kg} \mathrm{t}^{-1}\right), \mathrm{K}\left(4.20\right.$ to $\left.4.42 \mathrm{~kg} \mathrm{t}^{-1}\right), \mathrm{Mg}\left(0.34\right.$ to $\left.0.35 \mathrm{~kg} \mathrm{t}^{-1}\right)$ and Fe (10.18 to $\left.10.25 \mathrm{~g} \mathrm{t}^{-1}\right)$, while in Nayarit it was for $\mathrm{B}\left(13.25 \mathrm{~g} \mathrm{t}^{-1}\right)$.

Index words: Persea americana Miller, ecophysiology, mesocarp, mineral nutrition.

\section{INTRODUCCIÓN}

La planta de aguacate recicla (reabsorbe) una proporción importante de nutrimentos previo a la abscisión de sus hojas (Salazar-García et al., 2007); sin embargo, los nutrimentos removidos (sacados del huerto) por la cosecha representan una pérdida de nutrientes del suelo (Palmer y Dryden, 2006). El conocer la cantidad de nutrimentos removidos por el fruto es necesario para elaborar programas de fertilización que resulten en máximos rendimientos de fruto de calidad comercial (Salazar-García, 2002).

La remoción de nutrimentos puede ser influenciada por diversos factores. En Michoacán fue mayor la remoción de $\mathrm{N}$ y Ca por aguacate Hass en clima templado subhúmedo que en semicálido subhúmedo y semicálido húmedo (Salazar-García e Ibarra-Estrada, 2017). No se encontró influencia del riego sobre la remoción de nutrimentos; sin embargo, la época de floración sí tuvo efecto. Los frutos provenientes de la floración "marceña" (febrero-marzo) removieron mayor cantidad de N, K, Ca, Mg, S, Fe, Cu y 
Mn, en comparación con los de las floraciones "normal" (diciembre-febrero) y "loca" (agosto-septiembre).

El cv. Méndez ha adquirido importancia económica en México porque su cosecha principal se realiza entre junio y septiembre, cuando hay producción baja de Hass. Se estima que en Michoacán existen 10,000 ha con Méndez, 8,000 en Jalisco y 1500 en Nayarit (Gómez-Arreguí, 2018; Com. Pers. $)^{\text {. }}$.

Aunque existen diferencias entre el comportamiento fenológico de Hass (Cossio-Vargas et al., 2008; RochaArroyo et al., 2011) y de Méndez (Salazar-García et al., 2018), su fertilización se maneja como la de Hass. En Michoacán se encontró que, con excepción de N, P, Cu y $B$, el cv. Méndez remueve más nutrimentos que Hass (Salazar-García et al., 2016). Debido a que el estado nutrimental de los frutos de aguacate es consecuencia de factores edáficos, climáticos, manejo agronómico, nutrición, variedad y época de floración, que interaccionan en una zona productora, el objetivo de esta investigación fue comparar la concentración y remoción de N, P, K, Ca, $\mathrm{Mg}, \mathrm{S}, \mathrm{Fe}, \mathrm{Cu}, \mathrm{Mn}, \mathrm{Zn}$ y B por los tejidos del fruto (exocarpio o piel, mesocarpio o pulpa, cubierta seminal y embrión) de

'Gómez-Arreguí I. (2018) Dirección de la Asociación de Productores Exportadores de Aguacate de Jalisco, Ciudad Guzmán, Jalisco, México. la producción principal (junio a septiembre) del aguacate Méndez cultivado en las regiones de Jalisco, Michoacán y Nayarit.

\section{MATERIALES Y MÉTODOS}

\section{Características de las regiones}

Se seleccionaron siete huertos comerciales de aguacate Méndez con fertirriego, dos en el estado de Jalisco, cuatro en Michoacán y uno en Nayarit. La ubicación geográfica de los huertos, el tipo de suelo y el clima de cada región, bajo el sistema de clasificación de Köppen modificado por García (1998), se señalan en el Cuadro 1.

\section{Muestreo}

En cada huerto se escogieron 10 árboles cuya edad varió de 7 a 8 años, con una altura no mayor de $6 \mathrm{~m}$, sin entrecruzamiento de copas y con las siguientes producciones promedio de los dos años previos al estudio (suma de las cosechas de las floraciones de invierno y verano; datos proporcionados por el productor cooperante de cada huerto): Jalisco 52 kg (Colorín-1 y Ocote Cuate-2); Michoacán $45 \mathrm{~kg}$ (Cerritos), $35 \mathrm{~kg}$ (Manantiales), $48 \mathrm{~kg}$ (Sesángari) y $60 \mathrm{~kg}$ (El Colorín); Nayarit 52 kg (El Parejo). En todos los huertos el manejo lo realizó el productor

Cuadro 1. Nombre del huerto, ubicación, tipo de suelo y clima de las regiones de estudio.

\begin{tabular}{|c|c|c|c|}
\hline Huerto y ubicación & Latitud/longitud/altitud & Tipo de suelo & Tipo de clima \\
\hline $\begin{array}{l}\text { Colorín-1 } \\
\text { Zapotlán el Grande, Jalisco }\end{array}$ & $\begin{array}{l}19^{\circ} 42^{\prime} 57.1^{\prime \prime} \mathrm{N} \\
103^{\circ} 31^{\prime} 11.9^{\prime \prime} \mathrm{O} \\
1,556 \mathrm{~m}\end{array}$ & Feozem háplico & (A)C(wo) Semicálido subhúmedo \\
\hline $\begin{array}{l}\text { Ocote Cuate-2 } \\
\text { Zapotiltic, Jalisco }\end{array}$ & $\begin{array}{l}19^{\circ} 36^{\prime} 08.9^{\prime \prime} \mathrm{N} \\
103^{\circ} 27^{\prime} 00.6^{\prime \prime} \mathrm{O} \\
1,428 \mathrm{~m}\end{array}$ & Feozem háplico & (A)C(wo) Semicálido subhúmedo \\
\hline $\begin{array}{l}\text { Cerritos } \\
\text { Peribán, Michoacán }\end{array}$ & $\begin{array}{l}19^{\circ} 32^{\prime} 21.12^{\prime \prime} \mathrm{N} \\
102^{\circ} 26^{\prime} 16.15^{\prime \prime} \mathrm{O} \\
1,497 \mathrm{~m}\end{array}$ & Andosol ócrico & (A)C(w1) Semicálido subhúmedo \\
\hline $\begin{array}{l}\text { Manantiales } \\
\text { Uruapan, Michoacán }\end{array}$ & $\begin{array}{l}19^{\circ} 24^{\prime} 0.92^{\prime \prime} \mathrm{N} \\
102^{\circ} 04^{\prime} 31.18^{\prime \prime} \mathrm{O} \\
1,631 \mathrm{~m}\end{array}$ & Cambisol eútrico & (A)C(w2) Semicálido subhúmedo \\
\hline $\begin{array}{l}\text { Sesángari } \\
\text { Uruapan, Michoacán }\end{array}$ & $\begin{array}{l}19^{\circ} 24^{\prime} 23.15^{\prime \prime} \mathrm{N} \\
102^{\circ} 06^{\prime} 2.60^{\prime \prime} \mathrm{O} \\
1,809 \mathrm{~m}\end{array}$ & Cambisol eútrico & (A)C(w2) Semicálido subhúmedo \\
\hline $\begin{array}{l}\text { El Colorín } \\
\text { Uruapan, Michoacán }\end{array}$ & $\begin{array}{l}19^{\circ} 25^{\prime} 14.88^{\prime \prime} \mathrm{N} \\
102^{\circ} 01^{\prime} 49.24^{\prime \prime} \mathrm{O} \\
1,624 \mathrm{~m}\end{array}$ & Andosol húmico & C(m) Templado húmedo \\
\hline $\begin{array}{l}\text { El Parejo } \\
\text { Tepic, Nayarit }\end{array}$ & $\begin{array}{l}21^{\circ} 32^{\prime} 10.3^{\prime \prime} \mathrm{N} \\
104^{\circ} 54^{\prime} 54.1^{\prime \prime} \mathrm{O} \\
1,144 \mathrm{~m}\end{array}$ & Luvisol crómico & Aw2 Cálido subhúmedo \\
\hline
\end{tabular}


cooperante, según el Plan de Trabajo para la Exportación de Aguacate Hass (SENASICA, 2011).

\section{Fertilidad del suelo}

Teniendo en cuenta la homogeneidad en el manejo agronómico y la mínima pendiente del suelo, en cada huerto se eligieron al azar cinco de los árboles inicialmente seleccionados y de cada uno de ellos se obtuvieron cuatro submuestras de suelo equidistantes del área de goteo y de 0 a $30 \mathrm{~cm}$ de profundidad (zona de mayor presencia de raíces finas). Con las 20 submuestras de cada huerto se conformó una muestra compuesta que se envió para su análisis al laboratorio Fertilab (https://www.fertilab. com.mx/new/), acreditado en los Estados Unidos de Norteamérica en calidad analítica por el programa NAPT (North American Proficiency Testing) que coordina la Sociedad Americana de la Ciencia del Suelo. Se determinó la textura, pH (1:2 agua) (McLean, 1982), materia orgánica por el método de Walkley y Black (Nelson y Sommers, 1982), P-Bray (Bray y Kurtz, 1945), K, Ca, Mg y Na con acetato de amonio (Doll y Lucas, 1973), Fe, Zn, Cu, Mn por el método DTPA (Lindsay y Norvell, 1978) y B por el método de agua caliente y Azometina-H (Bingham, 1982). Con excepción del $\mathrm{P}$ y $\mathrm{B}$, los cuales se determinaron en un espectrofotómetro Genesis 20 (Thermo Scientific, Madison, Wisconsin, EUA), el resto de los elementos se cuantificaron con un espectrofotómetro de absorción atómica (Thermo Series S, Thermo Scientific, Madison, Wisconsin, EUA). La interpretación de los resultados se realizó de acuerdo con la Norma Oficial Mexicana NOM-021-RECNAT-2000 (SEMARNAT, 2002)

\section{Programas de fertilización}

El programa de fertilización fue diferente entre huertos (Cuadro 2), ya que con excepción de los huertos Cerritos, Manantiales y el Colorín de Michoacán, éste se diseñó para cubrir sus requerimientos nutrimentales, según lo planteado por Salazar-García (2002) y Salazar-García et al. (2009).
Durante la ausencia de lluvias (octubre-junio) se usó fertirriego empleando los fertilizantes solubles siguientes: fosfonitrato (33-3-0), MAP técnico (12-61-0), sulfato de potasio (0-0-50-18.5 S), nitrato de potasio (14-0-44-2 S), solubor DF (17.5 B), nitrato de magnesio (14 N, $21 \mathrm{Mg})$, nitrato de calcio $(15.5 \mathrm{~N}, 21 \mathrm{Mg})$, sulfato de manganeso (32 Mn, 12.9 S), MKP (0-52-34), sulfato de zinc (31 Zn, 13 S), sulfato de magnesio (16 Mg, 32 S) y ácido fosfórico (85 P). La frecuencia de aplicación fue de semanal a mensual, dependiendo del tipo de suelo y el criterio del productor cooperante. Durante el periodo de lluvias (julio-septiembre) se realizaron mensualmente aplicaciones directas al suelo de los siguientes fertilizantes: fosfonitrato (33-3-0), MAP granular (11-52-0), sulfato de potasio (0-0-50-26 S), sulfato de zinc (32 Zn, 23 S) y boronat (31 B).

\section{Cosecha de frutos}

Los frutos correspondieron a la floración de verano y se recolectaron cuando alcanzaron la madurez de cosecha, determinada por el contenido de materia seca en el mesocarpio $\geq 22.7 \%$, determinado en horno de microondas (Herrera-González et al., 2017). Las fechas de floración y cosecha fueron: Jalisco (agosto-septiembre 2014; junio 2015), Michoacán (septiembre 2014; julio 2015) y Nayarit (septiembre 2014; julio 2015). De la periferia de cada árbol se obtuvieron cinco frutos al azar, los cuales se separaron en sus tejidos (exocarpio o piel, mesocarpio o pulpa, cubierta seminal y embrión) y se determinó el peso de la materia fresca de cada tejido. Posteriormente, se secaron en un horno con aire forzado (Lab line 34887 Thomas Scientific, Madison, Wisconsin, EUA), a $70{ }^{\circ} \mathrm{C}$ durante 72 h hasta peso constante. Previo al análisis químico, se prepararon muestras compuestas por árbol para cada tejido del fruto. Cada muestra compuesta se conformó con los tejidos de los cinco frutos obtenidos de cada árbol, teniendo 10 muestras compuestas por huerto.

Cuadro 2. Nutrientes aplicados anualmente $\left(\mathrm{kg} \mathrm{ha}^{-1}\right)$ en los huertos donde se recolectó el fruto.

\begin{tabular}{lrrrrrrrrr}
\hline $\begin{array}{l}\text { Región/huerto/árboles por } \\
\text { hectárea }\end{array}$ & $\mathrm{N}$ & $\mathrm{P}_{2} \mathrm{O}_{5}$ & $\mathrm{~K}_{2} \mathrm{O}$ & $\mathrm{CaO}$ & $\mathrm{Mg}$ & $\mathrm{Fe}$ & $\mathrm{Mn}$ & $\mathrm{Zn}$ & $\mathrm{B}$ \\
\hline Jalisco/Ocote Cuate-2/400 & 151 & 62 & 166 & 118 & 11 & 1.5 & 1.6 & 1.8 & 4.3 \\
Jalisco/Colorín-1/408 & 97 & 38 & 120 & 5 & 18 & 1.2 & 1.1 & 0.9 & 3.2 \\
Michoacán/Cerritos/416 & 25 & 5 & 19 & 0 & 0 & 0.0 & 0.0 & 0.0 & 0.4 \\
Michoacán/Manantiales/555 & 150 & 137 & 225 & 524 & 343 & 2.5 & 0.3 & 0.3 & 0.2 \\
Michoacán/El Colorín/156 & 37 & 34 & 56 & 131 & 83 & 0.6 & 0.1 & 0.1 & 0.1 \\
Michoacán/Sesángari/666 & 158 & 75 & 94 & 28 & 11 & 0.0 & 0.0 & 0.1 & 0.1 \\
Nayarit/El Parejo/247 & 112 & 3 & 51 & 60 & 8 & 1.4 & 0.4 & 12.7 & 4.1 \\
\hline
\end{tabular}




\section{Análisis nutrimental del fruto}

Las muestras de cada tejido se analizaron en el laboratorio acreditado mencionado previamente. Se cuantificó en la materia seca la concentración de N, P, K, Ca, Mg, S, Fe, Cu, Mn, Zn y B. La determinación de N-total fue mediante el método semi-microKjeldahl (Alcántar-González y SandovalVilla, 1999; Bremner y Mulvaney, 1982), el cual consiste en la oxidación húmeda de la materia orgánica usando ácido sulfúrico y un catalizador, mientras que para $\mathrm{NO}_{3}{ }^{-}$se empleó el método de nitración con ácido salicílico (AlcántarGonzález y Sandoval-Villa, 1999). El K se extrajo con agua destilada y se cuantificó empleando un espectrofotómetro de absorción atómica (ICE 3000, Thermo Scientific, Madison, Wisconsin, EUA) (Alcántar-González y Sandoval-Villa, 1999). El P y S se determinaron utilizando los métodos de vanadatomolibdato amarillo y turbidimetría, respectivamente. Para el $\mathrm{Ca}, \mathrm{Mg}, \mathrm{Cu}, \mathrm{Fe}, \mathrm{Mn}$ y Zn se empleó el método de digestión en microondas $\mathrm{HNO}_{3}+\mathrm{HCl}$ (Alcántar-González y SandovalVilla, 1999). Todos estos nutrimentos se cuantificaron por absorción atómica empleando un espectrofotómetro ICAP 7200 (Thermo Scientific, Madison, Wisconsin, EUA). El B se determinó por el método de calcinación azometina-H (Enríquez, 1989) con un espectrofotómetro Genesis 20 (Thermo Scientific).

\section{Remoción de nutrimentos}

Los datos de concentración nutrimental se sometieron a control de calidad con el procedimiento Boxplot del programa computacional Minitab (Minitab Inc., 1996). La remoción de nutrimentos se determinó para cada tejido y posteriormente se sumaron para obtener la cantidad de nutrimentos removidos por tonelada de fruto fresco, de acuerdo con lo descrito por Mellado-Vázquez et al. (2012).

\section{Análisis estadístico}

Con la finalidad de comparar la concentración y remoción de los nutrimentos en los diferentes tejidos del fruto de aguacate por efecto de la región productora, se realizó análisis de varianza de una vía con el paquete estadístico SAS Ver. 9.3 (SAS Institute, 2011). La comparación de medias para determinar diferencias estadísticas se realizó con la prueba Waller-Duncan $(P \leq 0.05)$. También se calculó el coeficiente de correlación global de Pearson $(P \leq 0.05)$ entre la concentración de nutrientes en el suelo con la de cada tejido, considerando los datos de las tres regiones productoras como un solo conjunto de datos.

\section{RESULTADOS Y DISCUSIÓN}

\section{Fertilidad del suelo}

La fertilidad del suelo de los huertos varió entre regiones productoras (Cuadro 3). En Nayarit el pH del suelo fue moderadamente ácido, en Jalisco neutro y en Michoacán presentó valores moderadamente ácidos y neutros. El contenido de materia orgánica en Nayarit fue alto, en Michoacán varió de mediano a muy alto y en Jalisco de bajo a moderadamente bajo. Los suelos de Jalisco presentaron contenido moderadamente bajo de $\mathrm{Ca}, \mathrm{Mg}$ Mn y Zn. Al igual que los suelos de Jalisco, el de Nayarit presentó contenido moderadamente bajo de Ca y Mg, además de contenido bajo de Cu y B. En Michoacán el huerto Cerritos presentó contenidos moderadamente bajos de P, Ca y Cu y muy bajo de B. El huerto Manantiales tuvo bajo contenido de S y B. En el huerto Sesángari sólo el P estuvo moderadamente bajo. En el huerto El Colorín se observaron contenidos moderadamente bajos de Mn y B.

Los suelos de origen volcánico se caracterizan por contenido bajo de materia orgánica, Ca (Salazar-García, 2002) y micronutrientes (Salazar-García et al., 2016). Esta condición plantea la necesidad de aplicar fertilizantes orgánicos o inorgánicos para mejorar la fertilidad del suelo y satisfacer las necesidades del cultivo.

\section{Concentración de nutrimentos}

La concentración de algunos nutrimentos en los tejidos del fruto mostró diferencias entre regiones productoras (Cuadro 4). En el exocarpio, los frutos de Jalisco presentaron mayor concentración de P (0.12\%) y Cu (24.24 mg $\mathrm{kg}^{-1}$ ), mientras que los de Michoacán presentaron mayor concentración de K (1.20 \%). En el caso del Ca, la concentración fue superior en el exocarpio de los frutos de Jalisco y Michoacán (0.14 y $0.13 \%$ ), así como la de S y B en Michoacán (0.09\% y $38.52 \mathrm{mg} \mathrm{kg}^{-1}$ ) y Nayarit (0.08 \% y $44.98 \mathrm{mg} \mathrm{kg}^{-1}$ ).

El mesocarpio de los frutos de Michoacán presentó mayor concentración de S (0.22\%), Mn (9.0 mg kg-1) y B (70 $\mathrm{mg} \mathrm{kg}^{-1}$ ). De forma similar, el mesocarpio presentó mayor concentración de P, K, Mg y Cu en Michoacán (0.18, $1.86,0.13 \%$ y $12.40 \mathrm{mg} \mathrm{kg}^{-1}$, respectivamente) y Jalisco $\left(0.16,1.92,0.12 \%\right.$ y $\left.14.0 \mathrm{mg} \mathrm{kg}^{-1}\right)$.

En la cubierta seminal hubo coincidencia en la concentración mayor de $\mathrm{P}, \mathrm{Mn}, \mathrm{Zn}$ y B en Michoacán (0.17 \% y $80.0,50.7$ y $132.87 \mathrm{mg} \mathrm{kg}^{-1}$ ) y Nayarit (0.18\% y 94.63, 46.0 y $117.82 \mathrm{mg} \mathrm{kg}^{-1}$ respectivamente). En Jalisco la concentración de K (1.80 \%) fue superior. 
En el embrión, la concentración de nutrimentos mostró pocas diferencias entre zonas productoras. Se encontraron valores superiores de N (1.01\%) en Nayarit y de P, Mg, Fe y $\mathrm{Cu}\left(0.13,0.13 \%\right.$ y 40.55 y $\left.13.01 \mathrm{mg} \mathrm{kg}^{-1}\right)$ en Jalisco.

El clima de cada región estudiada es apto para el cultivo del aguacate Hass; sin embargo, las variaciones entre los climas y las diferencias en la fertilidad del suelo de los huertos pudieron provocar modificaciones en el crecimiento y por tanto en la concentración de nutrimentos en los frutos; aunado a ésto, las diferencias entre huertos en el manejo de la fertilización, principalmente con N, P y $\mathrm{K}$, que varió hasta en $100 \mathrm{~kg}$ entre huertos, así como la aplicación de $\mathrm{Ca}$, Mg y micronutrientes pudieron acentuar las diferencias encontradas en la concentración de nutrimentos en los frutos, ésto indica que la concentración de nutrimentos en el fruto, y por lo tanto su desarrollo y calidad tanto a la cosecha como en postcosecha, puede ser influenciada desde el huerto.
Salazar-García et al. (2011) estudiaron en Michoacán la influencia del tipo de clima, régimen de humedad del suelo y época de floración sobre la composición nutrimental del fruto de aguacate Hass y encontraron que la época de floración fue el factor que más afectó la concentración de K, Ca, Mg, S, Fe, Cu, Mn, Zn y B; sin embargo, también se encontraron que en el clima templado subhúmedo hubo mayores concentraciones de $\mathrm{N}$ y $\mathrm{Mn}$ en el exocarpio, de $\mathrm{N}, \mathrm{P}, \mathrm{K}, \mathrm{Ca}$ y $\mathrm{Mg}$ en el mesocarpio, de Mg, S, Cu y Mn en la cubierta seminal, y de B en la cubierta seminal y embrión. Los huertos sin riego mostraron mayores concentraciones de $\mathrm{Ca}$, S y Cu en el exocarpio, de P, K, Mn, Ca y Cu en el mesocarpio, de K, Mn y Cu en cubierta seminal, y de P, K, Mn en el embrión.

\section{Remoción de nutrimentos}

La remoción de nutrimentos por los tejidos del fruto de Méndez mostró diferencias entre regiones productoras (Cuadro 5). En el caso del exocarpio, los frutos de Jalisco

Cuadro 3. Fertilidad del suelo de los huertos de estudio.

\begin{tabular}{|c|c|c|c|c|c|c|c|c|c|c|c|}
\hline \multirow{2}{*}{$\mathrm{pH}$} & \multirow{2}{*}{$\begin{array}{l}\text { MO } \\
(\%)\end{array}$} & \multicolumn{10}{|c|}{$\mathrm{mg} \mathrm{kg}^{-1}$} \\
\hline & & $\mathrm{P}$ & $\mathrm{K}$ & $\mathrm{Ca}$ & $\mathrm{Mg}$ & S & $\mathrm{Fe}$ & $\mathrm{Cu}$ & $\mathrm{Mn}$ & $\mathrm{Zn}$ & B \\
\hline & \multicolumn{11}{|c|}{ Colorín-1, Jalisco } \\
\hline 6.8 & 1.5 & 70.4 & 268 & 1029 & 84.3 & 17.6 & 36 & 2.3 & 2.1 & 1.3 & 17.6 \\
\hline \multirow[t]{2}{*}{ N } & B & MA & M & MoB & $\mathrm{B}$ & MoA & $A$ & $A$ & B & MoB & MoA \\
\hline & \multicolumn{11}{|c|}{ Ocote Cuate-2, Jalisco } \\
\hline 6.6 & 1.6 & 66.4 & 491 & 859 & 183 & 17.6 & 79.4 & 4 & 3.8 & 1.1 & 0.3 \\
\hline \multirow[t]{2}{*}{$\mathrm{N}$} & MoB & MA & MoA & MoB & MoB & MoA & MA & MA & B & MoB & $\mathrm{MB}$ \\
\hline & \multicolumn{11}{|c|}{ Cerritos, Michoacán } \\
\hline 6.0 & 2.6 & 16 & 512.6 & 1122.9 & 622.3 & 61.6 & 85.5 & 5 & 87.5 & 16.8 & 0.3 \\
\hline \multirow[t]{2}{*}{ Mac } & MoA & MoB & MoA & MoB & MoA & M & A & MoB & MA & MoA & $\mathrm{MB}$ \\
\hline & \multicolumn{11}{|c|}{ Manantiales, Michoacán } \\
\hline 7.0 & 2.3 & 24.3 & 739.3 & 2511.7 & 826.5 & 23.4 & 49 & 7.3 & 24.9 & 8.6 & 0.5 \\
\hline \multirow[t]{2}{*}{$\mathrm{N}$} & M & M & A & MoA & $A$ & B & MoA & M & MoA & M & $\mathrm{B}$ \\
\hline & \multicolumn{11}{|c|}{ Sesángari, Michoacán } \\
\hline 5.9 & 3.2 & 11.7 & 1374.3 & 2381.3 & 585.6 & 99.6 & 68.6 & 26.3 & 12.4 & 73.7 & 2.6 \\
\hline \multirow[t]{2}{*}{ Mac } & MoA & MoB & MA & M & MoA & M & MoA & A & M & MA & MoA \\
\hline & \multicolumn{11}{|c|}{ El Colorín, Michoacán } \\
\hline 6.3 & 6.1 & 23.9 & 775.6 & 2200.1 & 602.9 & 53.9 & 32.7 & 48.7 & 5 & 26 & 0.8 \\
\hline \multirow[t]{2}{*}{ Mac } & MA & M & A & M & MoA & M & M & MA & MoB & A & MoB \\
\hline & \multicolumn{11}{|c|}{ El Parejo, Nayarit } \\
\hline 5.8 & 3.3 & 82.8 & 853.7 & 1221.4 & 167.8 & 110.4 & 65 & 1 & 10.3 & 6.1 & 0.3 \\
\hline Mac & A & MA & A & MoB & MoB & $\mathrm{M}$ & MA & B & $M$ & $M$ & MB \\
\hline
\end{tabular}

MO: materia orgánica, A: alto, MoA: moderadamente alto, MA: muy alto, M: mediano, B: bajo, MoB: moderadamente bajo, MB: muy bajo, N: neutral Mac: moderadamente ácido. 
Cuadro 4. Concentración de nutrimentos en la materia seca de tejidos del fruto de aguacate Méndez de las regiones de Jalisco (Jal), Michoacán (Mich) y Nayarit (Nay).

\begin{tabular}{|c|c|c|c|c|c|c|c|c|c|c|c|}
\hline \multirow{2}{*}{ Región } & \multicolumn{6}{|c|}{$\%$} & \multicolumn{5}{|c|}{$\mathrm{mg} \mathrm{kg}^{-1}$} \\
\hline & $\mathrm{N}$ & $P$ & $K$ & $\mathrm{Ca}$ & $\mathrm{Mg}$ & $S$ & $\mathrm{Fe}$ & $\mathrm{Cu}$ & $\mathrm{Mn}$ & $\mathrm{Zn}$ & $B$ \\
\hline & \multicolumn{11}{|c|}{ Exocarpio } \\
\hline Jal & 0.96 & $0.12 \mathrm{a}$ & $0.88 b$ & $0.14 a$ & 0.11 & $0.05 b$ & 43.03 & $24.24 \mathrm{a}$ & 12.32 & 15.43 & $31.42 b$ \\
\hline Mich & 0.94 & $0.08 b$ & $1.20 \mathrm{a}$ & $0.13 a$ & 0.14 & $0.09 a$ & 43.53 & $18.64 \mathrm{~b}$ & 11.43 & 20.33 & $38.52 \mathrm{a}$ \\
\hline Nay & 1.08 & $0.09 b$ & $0.87 b$ & $0.08 b$ & 0.08 & 0.08 a & 36.81 & $10.62 \mathrm{c}$ & 13.85 & 20.82 & $44.98 \mathrm{a}$ \\
\hline \multirow[t]{2}{*}{$\operatorname{Pr}>F$} & 0.108 & 0.003 & $<0.0001$ & 0.012 & 0.059 & $<0.0001$ & 0.215 & 0.0003 & 0.685 & 0.057 & 0.002 \\
\hline & \multicolumn{11}{|c|}{ Mesocarpio } \\
\hline Jal & $1.28 \mathrm{a}$ & $0.16 a$ & $1.92 \mathrm{a}$ & 0.17 & $0.12 a$ & $0.15 b$ & 33.98 & $14.00 \mathrm{a}$ & $5.00 \mathrm{c}$ & $21.23 b$ & $41.72 b$ \\
\hline Mich & $1.11 \mathrm{~b}$ & $0.18 a$ & $1.86 \mathrm{a}$ & 0.10 & $0.13 a$ & $0.22 \mathrm{a}$ & 37.13 & $12.40 \mathrm{a}$ & $9.00 \mathrm{a}$ & $26.10 \mathrm{a}$ & $70.00 \mathrm{a}$ \\
\hline Nay & $1.28 \mathrm{a}$ & $0.11 b$ & $1.52 b$ & 0.05 & $0.10 b$ & $0.11 \mathrm{c}$ & 34.23 & $7.21 \mathrm{~b}$ & $7.00 \mathrm{~b}$ & $\begin{array}{c}24.42 \\
a b\end{array}$ & $32.13 b$ \\
\hline \multirow[t]{2}{*}{$\operatorname{Pr}>\mathrm{F}$} & 0.021 & $<0.0001$ & $<0.0001$ & 0.162 & $<0.0001$ & $<0.0001$ & 0.604 & $<0.0001$ & $<0.0001$ & 0.023 & $<0.0001$ \\
\hline & \multicolumn{11}{|c|}{ Cubierta seminal } \\
\hline Jal & $1.41 \mathrm{~b}$ & $0.13 b$ & $1.80 \mathrm{a}$ & $0.26 \mathrm{a}$ & $0.34 \mathrm{a}$ & $0.10 b$ & 73.33 a & $26.60 \mathrm{a}$ & $38.50 \mathrm{~b}$ & $34.00 \mathrm{~b}$ & $62.76 b$ \\
\hline Mich & $1.43 b$ & $0.17 \mathrm{a}$ & $1.60 \mathrm{~b}$ & $0.24 a$ & $0.42 \mathrm{a}$ & $0.15 a$ & $73.95 \mathrm{a}$ & $21.40 \mathrm{~b}$ & $80.00 \mathrm{a}$ & $50.70 \mathrm{a}$ & $132.87 \mathrm{a}$ \\
\hline Nay & $1.73 \mathrm{a}$ & $0.18 a$ & $1.40 \mathrm{C}$ & $0.17 b$ & $0.35 a$ & $0.07 b$ & $48.30 \mathrm{~b}$ & $23.31 \mathrm{ab}$ & 94.63 a & $46.00 \mathrm{a}$ & $117.82 \mathrm{a}$ \\
\hline \multirow[t]{2}{*}{$\operatorname{Pr}>\mathrm{F}$} & 0.008 & $<0.0001$ & 0.002 & 0.016 & 0.029 & 0.0005 & 0.021 & 0.032 & $<0.0001$ & 0.001 & $<0.0001$ \\
\hline & \multicolumn{11}{|c|}{ Embrión } \\
\hline Jal & $0.81 b$ & $0.13 a$ & 0.87 & $0.12 \mathrm{a}$ & $0.13 a$ & 0.10 & $40.55 a$ & $13.10 \mathrm{a}$ & 8.60 & 28.00 & 15.00 \\
\hline Mich & $0.80 \mathrm{~b}$ & $0.10 b$ & 0.93 & $0.11 \mathrm{a}$ & $0.09 b$ & 0.11 & $27.44 b$ & 9.20 b & 7.20 & 19.00 & 16.05 \\
\hline Nay & $1.01 \mathrm{a}$ & $0.07 \mathrm{c}$ & 0.85 & $0.05 b$ & $0.06 \mathrm{~b}$ & 0.10 & $29.63 b$ & $4.24 \mathrm{c}$ & 4.00 & 16.00 & 19.11 \\
\hline $\operatorname{Pr}>\mathrm{F}$ & 0.003 & 0.0002 & 0.084 & 0.004 & 0.004 & 0.134 & 0.011 & $<0.0001$ & 0.121 & 0.412 & 0.173 \\
\hline
\end{tabular}

Medias con la misma letra en columnas, para cada tejido, son estadísticamente iguales (Waller-Duncan, $\mathrm{P} \leq 0.05$ ).

removieron mayor cantidad de P, Ca, Fe y Cu. El mesocarpio de los frutos de Jalisco y Michoacán coincidió en la mayor remoción de K, Mg y Cu. Respecto a la cubierta seminal, en los frutos de Jalisco ocurrió mayor remoción de K, Ca y Cu, mientras que en los de Nayarit la remoción generalmente fue más baja que en las otras dos regiones productoras. En el caso del embrión, con excepción del Zn, donde no hubo diferencias entre regiones, los frutos de Jalisco y Michoacán presentaron mayor remoción de nutrimentos que los de Nayarit.

La remoción total de nutrimentos por el fruto también mostró diferencias entre regiones productoras de Méndez (Cuadro 6). En Michoacán y Jalisco se presentó la mayor remoción total de $\mathrm{P}\left(0.41\right.$ a $\left.0.43 \mathrm{~kg} \mathrm{t}^{-1}\right), \mathrm{K}\left(4.20\right.$ a $\left.4.42 \mathrm{~kg} \mathrm{t}^{-1}\right)$, $\mathrm{Mg}\left(0.34\right.$ a $\left.0.35 \mathrm{~kg} \mathrm{t}^{-1}\right)$ y Fe (10.18 a $\left.10.25 \mathrm{~g} \mathrm{t}^{-1}\right)$; asimismo, los frutos de Jalisco removieron más $\mathrm{Cu}\left(4.20 \mathrm{~g} \mathrm{t}^{-1}\right)$ y los de Michoacán más S y Mn $\left(0.50 \mathrm{~kg} \mathrm{t}^{-1}\right.$ y $\left.2.57 \mathrm{~g} \mathrm{t}^{-1}\right)$. En Nayarit el nutrimento que mostró la mayor remoción total fue $\mathrm{B}$ $\left(13.25 \mathrm{~g} \mathrm{t}^{-1}\right)$.
Dos de los nutrimentos importantes para la nutrición del aguacate son $\mathrm{N}$ y $\mathrm{Zn}$; sin embargo, no mostraron diferencias entre regiones productoras. Esto ocurrió a pesar de la variación en la fertilización de cada huerto, que varió de 25 a $158 \mathrm{~kg} \mathrm{ha}^{-1}$ de N y de 0 a $12.7 \mathrm{~kg} \mathrm{ha}^{-1} \mathrm{de} Z \mathrm{n}$.

Diferencias en la cantidad de nutrimentos removidos por la cosecha entre regiones productoras no sólo ocurren en aguacate Méndez, también se han documentado en Hass. En Nayarit México, (clima cálido y suelo cambisol crómico), la remoción en $\mathrm{kg} \mathrm{t}^{-1}$ fue de $2.5 \mathrm{~N}, 1.0 \mathrm{P}, 3.8 \mathrm{~K}, 0.08 \mathrm{Ca}, 0.29$ $\mathrm{Mg}, 0.34 \mathrm{~S}$; y en $\mathrm{g} \mathrm{t}^{-1}$ de $6 \mathrm{Fe}, 2 \mathrm{Cu}, 1 \mathrm{Mn}, 4 \mathrm{Zn}$ y $0.2 \mathrm{~B}$ (SalazarGarcía y Lazcano-Ferrat, 2001). En California EUA (clima mediterráneo y suelo granítico), se menciona la siguiente remoción en $\mathrm{kg} \mathrm{t}^{-1}: 2.8 \mathrm{~N}, 1.05 \mathrm{P}, 6.7 \mathrm{~K}, 0.5 \mathrm{Ca}, 1.1 \mathrm{Mg}, 2 \mathrm{~S}, \mathrm{y}$ en $\mathrm{gt}^{-1}$ de 11.7 Fe, 14.4 Cu, 2.2 Mn, 38.6 Zn y 99.3 B (Hofshi y Hofshi, 2003). En el caso de Michoacán México, (climas semicálido y templado; suelos luvisol, cambisol y andosol), los frutos de la floración principal removieron en $\mathrm{kg} \mathrm{t}^{-1}: 2.4$ $\mathrm{N}, 0.5 \mathrm{P}, 3.3 \mathrm{~K}, 0.1 \mathrm{Ca}, 0.26 \mathrm{Mg}, 0.2 \mathrm{~S}$ en $\mathrm{g} \mathrm{t}^{-1} 6.4 \mathrm{Fe}, 3.3$ Cu, 1.6 Mn, 3.9 Zn y 8.0 B (Salazar-García e Ibarra-Estrada, 
Cuadro 5. Remoción de nutrimentos por los tejidos del fruto de aguacate Méndez de las regiones de Jalisco (Jal), Michoacán (Mich) y Nayarit (Nay).

\begin{tabular}{|c|c|c|c|c|c|c|c|c|c|c|c|}
\hline \multirow{2}{*}{ Región } & \multicolumn{6}{|c|}{$\mathrm{kg} \mathrm{t}^{-1}$} & \multicolumn{5}{|c|}{$\mathrm{g} \mathrm{t}^{-1}$} \\
\hline & $\mathrm{N}$ & $P$ & K & $\mathrm{Ca}$ & $\mathrm{Mg}$ & $S$ & $\mathrm{Fe}$ & $\mathrm{Cu}$ & $\mathrm{Mn}$ & $\mathrm{Zn}$ & B \\
\hline & \multicolumn{11}{|c|}{ Exocarpio } \\
\hline Jal & 0.33 a & $0.04 \mathrm{a}$ & $0.30 a b$ & $0.05 a$ & $0.04 \mathrm{a}$ & $0.01 \mathrm{~b}$ & $1.60 \mathrm{a}$ & $0.82 \mathrm{a}$ & 0.42 & 0.52 & 1.10 \\
\hline Mich & $0.27 b$ & $0.02 b$ & $0.34 a$ & $0.04 \mathrm{~b}$ & $0.04 a$ & $0.03 a$ & $1.24 b$ & $0.53 b$ & 0.32 & 0.60 & 1.10 \\
\hline Nay & $0.31 a b$ & $0.02 \mathrm{~b}$ & $0.26 \mathrm{~b}$ & $0.02 \mathrm{c}$ & $0.03 \mathrm{~b}$ & $0.02 \mathrm{a}$ & $1.10 \mathrm{~b}$ & $0.32 \mathrm{c}$ & 0.40 & 0.61 & 1.35 \\
\hline \multirow[t]{2}{*}{$\mathrm{Pr}>\mathrm{F}$} & 0.01 & 0.0003 & 0.026 & 0.0002 & 0.016 & 0.005 & 0.012 & $<0.0001$ & 0.272 & 0.555 & 0.112 \\
\hline & \multicolumn{11}{|c|}{ Mesocarpio } \\
\hline Jal & 2.13 & $0.30 \mathrm{~b}$ & $3.23 \mathrm{a}$ & 0.30 & $0.21 \mathrm{a}$ & $0.30 \mathrm{~b}$ & 6.00 & $2.40 \mathrm{a}$ & $0.75 c$ & $3.60 \mathrm{~b}$ & $7.00 \mathrm{~b}$ \\
\hline Mich & 2.00 & $0.32 \mathrm{a}$ & $3.28 \mathrm{a}$ & 0.18 & $0.23 a$ & $0.40 \mathrm{a}$ & 7.00 & $2.20 \mathrm{a}$ & $1.60 \mathrm{a}$ & 4.62 a & $6.00 \mathrm{~b}$ \\
\hline Nay & 2.10 & $0.20 \mathrm{c}$ & $2.50 \mathrm{~b}$ & 0.10 & $0.16 b$ & $0.20 \mathrm{c}$ & 6.00 & $1.20 \mathrm{~b}$ & $1.11 \mathrm{~b}$ & $4.00 a b$ & $11.30 \mathrm{a}$ \\
\hline \multirow[t]{2}{*}{$\mathrm{Pr}>\mathrm{F}$} & 0.257 & $<0.0001$ & $<0.0001$ & 0.186 & $<0.0001$ & $<0.0001$ & 0.225 & $<0.0001$ & $<0.0001$ & 0.009 & $<0.0001$ \\
\hline & \multicolumn{11}{|c|}{ Cubierta seminal } \\
\hline Jal & $0.03 \mathrm{a}$ & $0.002 \mathrm{a}$ & $0.04 \mathrm{a}$ & $0.005 a$ & $0.007 \mathrm{a}$ & 0.002 & $0.20 \mathrm{a}$ & $0.06 a$ & 0.08 & $0.10 \mathrm{a}$ & $0.13 a b$ \\
\hline Mich & $0.02 \mathrm{ab}$ & $0.002 \mathrm{a}$ & $0.02 b$ & $0.003 b$ & 0.006 a & 0.002 & $0.11 \mathrm{a}$ & $0.03 b$ & 0.12 & $0.10 a$ & $0.20 \mathrm{a}$ \\
\hline Nay & $0.01 \mathrm{~b}$ & $0.001 \mathrm{~b}$ & $0.01 \mathrm{c}$ & $0.001 \mathrm{c}$ & $0.002 b$ & 0.001 & $0.04 \mathrm{~b}$ & $0.02 \mathrm{~b}$ & 0.07 & $0.03 \mathrm{~b}$ & $0.08 \mathrm{~b}$ \\
\hline \multirow[t]{2}{*}{$\mathrm{Pr}>\mathrm{F}$} & 0.009 & 0.022 & 0.0001 & $<0.0001$ & 0.002 & 0.066 & 0.001 & 0.001 & 0.047 & 0.007 & 0.003 \\
\hline & \multicolumn{11}{|l|}{ Embrión } \\
\hline Jal & 0.60 a & 0.09 a & $0.64 \mathrm{a}$ & $0.09 a$ & 0.09 a & $0.07 \mathrm{a}$ & $3.00 \mathrm{a}$ & $1.00 \mathrm{a}$ & 0.63 a & 2.00 & $1.10 \mathrm{a}$ \\
\hline Mich & $0.65 a$ & $0.08 \mathrm{a}$ & $0.80 \mathrm{a}$ & $0.09 a$ & $0.07 \mathrm{a}$ & $0.09 \mathrm{a}$ & $2.30 \mathrm{a}$ & $1.00 \mathrm{a}$ & $0.60 \mathrm{a}$ & 1.50 & $1.31 \mathrm{a}$ \\
\hline Nay & $0.30 \mathrm{~b}$ & 0.02 b & 0.24 b & $0.01 b$ & $0.02 b$ & 0.02 b & $1.00 \mathrm{~b}$ & $0.12 b$ & $0.12 b$ & 0.50 & $0.54 b$ \\
\hline $\mathrm{Pr}>\mathrm{F}$ & 0.0004 & $<0.0001$ & $<0.0001$ & 0.0001 & 0.0007 & 0.0009 & 0.001 & $<0.0001$ & 0.023 & 0.107 & 0.001 \\
\hline
\end{tabular}

Medias con la misma letra en columnas, para cada tejido, son estadísticamente iguales (Waller-Duncan, $\mathrm{P} \leq 0.05$ ).

2017). La información disponible muestra que la remoción de nutrimentos es significativamente diferente entre regiones productoras. En todos los casos la remoción de nutrimentos por los frutos de California fue mayor. A pesar de las variaciones en las características de suelo y clima, así como de manejo de los huertos de Méndez (en este estudio) y Hass, el único nutrimento cuya remoción no fue notoriamente afectada por las condiciones de cultivo fue el nitrógeno, aspecto que requiere de mayor investigación.

En el presente estudio la remoción de nutrimentos por el fruto de aguacate Méndez fue mayor a lo reportado para otros cultivares de aguacate. Méndez removió mayor cantidad de N, K, Ca y B que los frutos de Hass de Nayarit (Salazar-García y Lazcano-Ferrat, 2001), de K, Ca, Mg, S, $\mathrm{Fe}, \mathrm{Mn}$ y Zn que Hass en Michoacán (Salazar-García et al., 2016). Esto mismo ocurrió con respecto a frutos de Hass de Antioquía, Colombia (Tamayo et al., 2018). La remoción de nutrimentos por Méndez también superó la de algunos cultivares de clima cálido en Nayarit, como fue el caso del $\mathrm{N}, \mathrm{Ca}, \mathrm{Mg}$ y S para el cv. Choquette, de N, K, Ca, Mg, Fe, Cu, Mn, Zn y B para el cv. Hall y de N, Zn y B para el cv. Booth 8 (Salazar-García y Lazcano-Ferrat, 2001).

\section{Correlación entre la concentración de nutrientes en el suelo y el fruto}

Los nutrientes en el suelo presentaron correlaciones con la concentración de algunos nutrimentos en el fruto (Cuadro 7). Para el exocarpio, las correlaciones positivas ocurrieron con P y S y las negativas con Fe y B. En el caso del mesocarpio, hubo correlaciones positivas con Mg y Mn y negativa con P y K. Witney et al. (1990) mencionaron que entre los nutrimentos que afectan la calidad postcosecha del fruto se encuentran el K y Mg. Por lo anterior, se deben mantener los niveles adecuados de estos nutrientes para evitar daños como oscurecimiento vascular. La cubierta seminal tuvo correlaciones positivas con $\mathrm{Mg}$ y $\mathrm{Mn}$ y negativas con Fe y B; esto último coincidió con el exocarpio.

Los niveles aceptables de algunos nutrientes del suelo pudieron disimular el efecto de la fertilización en la concentración de nutrimentos en los tejidos del fruto; tal es el caso del $\mathrm{K}$ y Fe, cuyos valores se reportaron entre medios y muy altos en el suelo de todos los huertos, y aunque la cantidad de fertilizante varió entre regiones, no se apreció una relación entre la fertilización y la concentración de 
Cuadro 6. Remoción total de nutrimentos por el fruto de aguacate Méndez de tres regiones productoras.

\begin{tabular}{|c|c|c|c|c|c|c|c|c|c|c|c|}
\hline \multirow{2}{*}{ Región } & \multicolumn{6}{|c|}{$\mathrm{kg} \mathrm{t}^{-1}$} & \multicolumn{5}{|c|}{$\mathrm{g} \mathrm{t}^{-1}$} \\
\hline & $\mathrm{N}$ & $P$ & $\mathrm{~K}$ & $\mathrm{Ca}$ & $\mathrm{Mg}$ & S & $\mathrm{Fe}$ & $\mathrm{Cu}$ & $\mathrm{Mn}$ & Zn & B \\
\hline Jalisco & 3.10 & $0.41 \mathrm{a}$ & $4.20 \mathrm{a}$ & $0.43 a$ & $0.34 \mathrm{a}$ & $0.34 \mathrm{~b}$ & $10.25 a$ & $4.20 \mathrm{a}$ & $1.83 \mathrm{~b}$ & 6.04 & $9.12 b$ \\
\hline Michoacán & 3.00 & $0.43 a$ & $4.42 \mathrm{a}$ & $0.31 \mathrm{ab}$ & $0.35 a$ & $0.50 \mathrm{a}$ & $10.18 \mathrm{a}$ & $3.50 \mathrm{~b}$ & $2.57 \mathrm{a}$ & 6.80 & $8.20 \mathrm{~b}$ \\
\hline Nayarit & 3.00 & $0.23 b$ & $3.00 \mathrm{~b}$ & $0.13 b$ & $0.21 \mathrm{~b}$ & $0.22 \mathrm{c}$ & $7.50 \mathrm{~b}$ & $1.62 \mathrm{c}$ & $1.70 \mathrm{~b}$ & 5.04 & $13.25 a$ \\
\hline $\operatorname{Pr}>\mathrm{F}$ & 0.178 & $<0.0001$ & $<0.0001$ & 0.043 & $<0.0001$ & $<0.0001$ & 0.021 & $<0.0001$ & 0.0001 & 0.184 & 0.0006 \\
\hline
\end{tabular}

Medias con la misma letra en columnas son estadísticamente iguales (Waller-Duncan, $\mathrm{P} \leq 0.05$ ).

Cuadro 7. Correlaciones globales significativas entre el contenido de nutrientes en el suelo y su concentración en algunos tejidos del fruto de aguacate Méndez cosechados en verano de 2015 en Jalisco, Michoacán y Nayarit, México.

\begin{tabular}{lcc}
\hline Tejido & Elemento & Correlación \\
\hline Exocarpio & $\mathrm{P}$ & $0.31 *$ \\
& $\mathrm{~S}$ & $0.41 * *$ \\
& $\mathrm{Fe}$ & $-0.43^{* *}$ \\
& $\mathrm{~B}$ & $-0.31 *$ \\
Mesocarpio & & \\
& $\mathrm{P}$ & $-0.49 * *$ \\
& $\mathrm{~K}$ & $-0.35 *$ \\
& $\mathrm{Mg}$ & $0.39 * *$ \\
& $\mathrm{Mn}$ & $0.59 * *$ \\
Cubierta seminal & $\mathrm{Mg}$ & $0.42 * *$ \\
& $\mathrm{Fe}$ & $-0.63 * *$ \\
$\mathrm{Mn}$ & $0.48 * *$ \\
$\mathrm{~B}$ & $-0.52 * *$ \\
\hline
\end{tabular}

* $\mathrm{y} * *$ : significativo con $\mathrm{P} \leq 0.05 \mathrm{y} P \leq 0.01$, respectivamente.

estos nutrimentos en los tejidos. En Michoacán $96 \%$ de los huertos de aguacate Hass evaluados por MaldonadoTorres et al. (2007) mostraron de altos a muy altos contenidos de Fe y $\mathrm{K}$ en el suelo; tal pareciera que, a nivel general, en las principales zonas productoras de aguacate se cumplen los requerimientos de estos nutrimentos.

Por otra parte, los contenidos bajos a muy bajos de algunos nutrientes en el suelo, como Mg, Mn, Zn y B en Jalisco pudieron cambiar el destino de los nutrientes aportados por la fertilización hacia órganos de mayor demanda que el fruto, lo que pudiera explicar las bajas concentraciones de algunos nutrimentos en los tejidos evaluados.

\section{CONCLUSIONES}

La concentración de la mayoría de los nutrimentos en los tejidos del fruto de aguacate Méndez varió entre las regiones de producción incluidas en este estudio; sin embargo, el embrión mostró menos diferencias. La remoción total de nutrimentos por tonelada de fruto también presentó diferencias entre regiones productoras; el fruto de Nayarit tuvo menor remoción que el de Jalisco y Michoacán. Con excepción de $\mathrm{N}$ y Zn, la región de producción afectó la remoción total de nutrimentos.

\section{AGRADECIMIENTOS}

Se reconoce el financiamiento del INIFAP y de Agro Gonzámex (Jalisco). Los autores agradecen el apoyo de productores de la APEAM (Michoacán), Agro González S.P.R. de R.L. (Jalisco) y a PARGUT S.P.R. de R.L. (Nayarit) por proporcionar los frutos para esta investigación.

\section{BIBLIOGRAFÍA}

Alcántar-González G. y M. Sandoval-Villa (1999) Manual de Análisis Químico de Tejido Vegetal. Guía de Muestreo, Preparación, Análisis e Interpretación. Publicación Especial Núm. 10 Sociedad Mexicana de la Ciencia del Suelo, A. C. Chapingo, Estado de México. 155 p.

Bingham F. T. (1982) Boron. In: Methods of Soil Analysis: Part 2 Chemical and Microbiological Properties. A. L. Page, R. H. Miller and D. R. Keeney (eds.). American Society of Agronomy and Soil Science Society of America. Madison, Wisconsin, USA. pp:431-447, https://doi.org/10.2134/agronmonogr9.2.2ed.c25

Bray R. H. and L. T. Kurtz (1945) Determination of total, organic and available phosphorus in soil. Soil Science 59:39-45.

Bremner J. M. and C. S. Mulvaney (1982) Nitrogen-total. In: Methods of Soil Analysis: Part 2 Chemical and Microbiological Properties. 2nd. edition. A. L. Page, R. H. Miller, and D. R. Keeney (eds.). American Society of Agronomy and Soil Science Society of America. Madison, Wisconsin, USA. pp:595-617, https://doi. org/10.2134/agronmonogr9.2.2ed.c31

Cossio-Vargas L. E., S. Salazar-García, I. J. L. González-Durán y R. MedinaTorres (2008) Fenología del aguacate 'Hass' en el clima semicálido de Nayarit, México. Revista Chapingo Serie Horticultura 14:325-330.

Doll E. C. and R. E. Lucas (1973) Testing soil for potassium, calcium and magnesium. In: Soil Testing and Plant Analysis. L. M. Walsh and J. D. Beaton (eds.). Soil Science Society of America. Madison, Wisconsin, USA. pp:133-152.

Enríquez R. S. (1989) Análisis de boro en suelos y plantas mediante el método de azometina-H. Terra 7:13-20. 
García E. 1998. Comisión Nacional para el estudio de la Biodiversidad (CONABIO). Cartas de clima de la República Mexicana. Modificaciones al sistema de clasificación de Köppen. Escala 1:1000 000 México. Shapefile. http://www.conabio.gob.mx/ informacion/metadata/gis/clima1 mgw.xml?_httpcache=yes\& $x \mathrm{~s}=/ \mathrm{db} / \mathrm{metadata} / \mathrm{xs} / \mathrm{fgdc} \_$html.xsl\&_indent=no (Mayo 2021).

Herrera-González J. A., S. Salazar-García, H. E. Martínez-Flores y J. E. RuizGarcía (2017) Indicadores preliminares de madurez fisiológica y comportamiento postcosecha del fruto de aguacate Méndez. Revista Fitotecnia Mexicana 40:55-63, https://doi. org/10.35196/rfm.2017.1.55-63

Hofshi R. and S. Hofshi (2003) Total fruit nutrient removal calculator for Hass avocado in California. The Hofshi Foundation. Fallbrook, California, USA. http://www.avocadosource.com/tools/ nutremcalc.htm (Mayo 2021)

Lindsay W. L. and W. A. Norvell (1978) Development of a DTPA soil test for zinc, iron, manganese, and copper. Soil Science Society of America Journal 42:421-428, https://doi.org/10.2136/ sssaj1978.03615995004200030009x

Maldonado-Torres R., M. E. Álvarez-Sánchez, G. Almaguer-Vargas, A. F. Barrientos-Priego y R. García-Mateos (2007) Estándares nutrimentales para aguacatero 'Hass'. Revista Chapingo Serie Horticultura 13:103-108, https://doi.org/10.5154/r. rchsh.2006.11.051

McLean E. O. (1982) Soil pH and lime requirement. In: Methods of Soil Analysis: Part 2 Chemical and Microbiological Properties. 2nd. edition. A. L. Page, R. H. Miller and D. R. Keeney (eds.) American Society of Agronomy and Soil Science Society of America. Madison, Wisconsin, USA. pp:199-224, https://doi. org/10.2134/agronmonogr9.2.2ed.c12

Mellado-Vázquez A., S. Salazar-García, C. A. Treviño de la Fuente, I. J. L. González-Durán y A. López-Jiménez (2012) Composición y remoción nutrimental de frutos de mango 'Haden' y 'Tommy Atkins' bajo producción forzada. Revista Mexicana de Ciencias Agrícolas 3:925-941, https://doi.org/10.29312/remexca. v3i5. 1384

Minitab Inc. (1996) Minitab for Windows, Release 11.2. State College, Pennsylvania, U.S.A.

Nelson D. W. and L. E. Sommers (1982) Total carbon, organic carbon and organic matter. In: Methods of Soil Analysis: Part 2 Chemical and Microbiological Properties. 2nd. edition. A. L. Page, R. H. Miller and D. R. Keeney (eds.). American Society of Agronomy and Soil Science Society of America. Madison, Wisconsin, USA. pp:539-579, https://doi.org/10.2134/agronmonogr9.2.2ed.c29

Palmer J. W. and G. Dryden (2006) Fruit mineral removal rates from New Zealand apple (Malus domestica) orchards in the Nelson region, New Zealand. Journal of Crop and Horticultural Science 34:2732, https://doi.org/10.1080/01140671.2006.9514384

Rocha-Arroyo J. L., S. Salazar-García, A. E. Bárcenas-Ortega, I. J. L. GonzálezDurán y L. E. Cossio-Vargas (2011) Fenología del aguacate 'Hass' en Michoacán. Revista Mexicana de Ciencias Agrícolas 2:303316.

Salazar-García S. (2002) Nutrición del Aguacate, Principios y Aplicaciones. Instituto Nacional de Investigaciones Forestales, Agrícolas y Pecuarias e Instituto de la Potasa y el Fósforo. Querétaro, México. 165 p.

Salazar-García S., A. Mellado-Vázquez, J. A. Herrera-González y A. ÁlvarezBravo (2016) Diferencias en la remoción de nutrimentos por el fruto de 'Hass' y 'Méndez' en Michoacán. In: Innovando el Agro Veracruzano. Frente a los Retos de la Relación SociedadNaturaleza. F. Gallardo-López (ed.). Colegio de Postgraduados. Veracruz, México. pp:242-256.

Salazar-García, S. and I. Lazcano-Ferrat (2001) Identifying fruit mineral removal differences in four avocado cultivars. Better Crops International 15:28-31.

Salazar-García S., I. J. L. González-Durán y L. M. Tapia-Vargas (2011) Influencia del clima, humedad del suelo y época de floración sobre la biomasa y composición nutrimental de frutos de aguacate 'Hass' en Michoacán, México. Revista Chapingo Serie Horticultura 17:183-194.

Salazar-García S., L. E. Cossio-Vargas e I. J. L. González-Durán (2007) Reciclamiento de nutrimentos por las hojas de aguacate 'Hass'. In: Actas VI Congreso Mundial de la Palta-Aguacate-Avocado. 12-16 de Noviembre de 2007. Viña del Mar, Chile. 11 p. http:// www.avocadosource.com/wac6/es/extenso/3a-102.pdf (Noviembre 2017).

Salazar-García, S., L. E. Cossio-Vargas e I. J. L. González-Durán (2009) La fertilización de sitio específico mejoró la productividad del aguacate 'Hass' en huertos sin riego. Agricultura Técnica en México 35:439-448.

Salazar-García S. y M. E. Ibarra-Estrada (2017) El tipo de clima, riego y época de floración afectó la remoción de nutrimentos por el fruto de aguacate 'Hass'. In: Aportaciones Científicas para la Horticultura Mexicana. Libro Científico No. 3. M. A. Urías-López, A. Álvarez-Bravo, L. M. Hernández-Fuentes y M. H. PérezBarraza (eds.). Campo Experimental Santiago Ixcuintla, INIFAP Santiago Ixcuintla, Nayarit, México. pp:95-98.

Salazar-García S., M. E. Ibarra-Estrada y R. Medina-Torres (2016) Tejidos alternativos al foliar para evaluar la respuesta a la fertilización con Zn o B en aguacate Hass. Revista Fitotecnia Mexicana 39:247-252, https://doi.org/10.35196/rfm.2016.3.247-252

Salazar-García, S., M. E. Ibarra-Estrada, M. E. y J. González-Valdivia (2018) Fenología del aguacate 'Méndez' en el sur de Jalisco, México Agrociencia 52:991-1003

SAS Institute (2011) SAS/STAT ${ }^{\oplus} 9.3$ User's Guide. Statistical Analysis System Institute Inc. Cary, North Crolina, USA. 8621 p.

SEMARNAT, Secretaría de Medio Ambiente y Recursos Naturales (2002) Norma Oficial Mexicana NOM-021-RECNAT-2000, Que establece las especificaciones de fertilidad, salinidad y clasificación de suelos. Estudios, muestreo y análisis. Diario Oficial de la Federación 31 de diciembre de 2002. México, D.F $73 \mathrm{p}$.

SENASICA, Servicio Nacional de Sanidad, Inocuidad y Calidad Agroalimentaria (2011) Plan de trabajo para la exportación de aguacate Hass de México a los Estados Unidos de América. Servicio Nacional de Sanidad, Inocuidad y Calidad Agroalimentaria. México, D. F. $32 \mathrm{p}$

Tamayo V. A., J. A. Bernal E. and C. A. Díaz D. (2018) Composition and removal of nutrients by the harvested fruit of avocado cv. Hass in Antioquia. Revista Facultad Nacional de Agronomía 71: 8511-8516, https://doi.org/10.15446/rfna.v71n2.71929

Witney G. W., P. J. Hofman and B. N. Wolstenholme (1990) Effect of cultivar, tree vigour and fruit position on calcium accumulation in avocado fruits. Scientia Horticulturae 44:269-278, https://doi org/10.1016/0304-4238(90)90127-Z 
